\title{
Intestinal Obstruction: Role of MDCT with Surgical Correlation
}

\author{
Amandeep Singh ${ }^{1}$, Indreet Kaur Makkar ${ }^{2}$, CL Thukral ${ }^{3}$, Kamlesh Gupta ${ }^{4}$, Manjit Singh Uppal ${ }^{5}$ \\ ${ }^{1}$ Associate Professor, Department of Radiodiagnosis \& Imaging, SGRD Institute of Medical Sciences and Research, Amritsar, India, ${ }^{2}$ Senior Resident, Department \\ of Radiodiagnosis \& Imaging, SGRD Institute of Medical Sciences And Research, Amritsar, India, ${ }^{3}$ Professor, Department of Radiodiagnosis \& Imaging, SGRD \\ Institute of Medical Sciences and Research, Amritsar, India, ${ }^{4}$ Professor \& Head, Department of Radiodiagnosis \& Imaging, SGRD Institute Of Medical Sciences And \\ Research, Amritsar, India, ${ }^{5}$ Director-Principal , Professor Surgery SGRD Institute of Medical Sciences \& Research, Amritsar, India.
}

\section{Abstract}

Background: To evaluate efficacy of CT (computed tomography) in diagnosis of the presence and cause of obstruction along with correlation of CT findings with the operative findings. Subjects and Methods: This prospective study included evaluation of forty patients, clinically suspected of intestinal obstruction by CT examination whose surgical follow up data was available. All patients (age $>18 y r s$ ) clinically suspected for intestinal obstruction were included. Results: Highly significant correlation was found between CT and operative findings in diagnosis of the cause of obstruction with $\mathrm{p}$ value of <0.01 and cohen's kappa value ranging from 0.8 to 1 (highly significant correlation). Conclusion: MDCT (Multi detector computed tomography) by using its multiplanar and 3D capabilities is highly accurate and specific in detecting the presence of intestinal obstruction and it can demonstrate the exact site of obstruction in high percent of cases, hence is helpful for appropriate treatment plan and management of the patient.

Keywords: Computed tomography; Small Bowel Obstruction; Hernia.

Corresponding Author: Dr. Amandeep Singh, Associate Professor, Department of Radiodiagnosis and Imaging, Sgrdimsar, Vallah, Amritsar, Punjab, India.

Received: December 2018

Accepted: December 2018

\section{Introduction}

Intestinal obstruction is one of the common cause of admission to emergency department with acute abdomen. The early diagnosis of bowel obstruction is critical in preventing complications, particularly perforation and ischemia. ${ }^{[1]}$

Small bowel obstruction remains important cause of morbidity accounting for upto $15 \%$ of surgical admissions for non - traumatic abdominal pain. ${ }^{[2]}$

Plain abdominal radiography continues to be the initial examination in these patients due to its wide availability and relatively low cost. However, radiographs are diagnostic in only $50 \%-60 \%$ of cases and have high sensitivity only for high-grade obstruction. Nevertheless, the results of this modality should serve as a basis for triage for further imaging work-up and assist in the therapeutic decision. ${ }^{[3-5]}$ Sonography is not commonly used for the evaluation of SBO (Small Bowel Obstruction) mainly because most of the time the bowel loops are filled with gas, producing nondiagnostic sonograms, and because adhesions, the most common cause of mechanical SBO, are not detected with this technique. ${ }^{[6]}$ However, when the obstructed bowel segments are dilated and filled with fluid, not only can the level of obstruction be recognized but the cause of the obstruction can also be demonstrated by using the fluidfilled bowel as a sonic window. ${ }^{[6,7]}$

Contrast material-enhanced studies, particularly volume- challenge enteral examinations like enteroclysis, were once advocated as the definitive study in patients with clinical uncertainty about the diagnosis of SBO, since these studies correctly demonstrate the presence of obstruction in $100 \%$ of cases, the level (proximal vs distal) of obstruction in $89 \%$ of cases, and the cause of the obstruction in $86 \%$ of surgically treated patients. ${ }^{[8]}$

The new technology, that is of increasing interest in the diagnosis of small bowel obstruction is multiplanar reformatted imaging at a workstation. Volume data of the abdomen is acquired with the helical technique during a single breath hold, usually with a collimation of $5 \mathrm{~mm}$. MDCT scanner enables better spatial resolution through thinner collimation. Axial, sagittal, coronal, and curved multiplanar reformatted images are created at a workstation from the acquired volume data. Multiplanar views may help identify the site, level and cause of obstruction when axial images are indeterminate. ${ }^{[9]}$

Conversely, if the initial radiographic findings are interpreted as normal, equivocal, or suggestive of a lowgrade partial SBO, an examination that challenges the distensibility of the small bowel such as small bowel follow-through study, enteroclysis, or CT enteroclysis is recommended, as these usually exaggerate the effects of mild obstructions. ${ }^{[3,10-13]}$ Nevertheless, we emphasize that a bowel obstruction is a dynamic and ever-changing process. It can rapidly evolve into a catastrophic condition with ischemia or resolve by itself. Therefore, in those cases where surgical treatment is not immediate or advocated, it is 
necessary to maintain close communication between the surgeon and radiologist in order to guarantee the appropriate imaging and clinical follow-up. ${ }^{[14]}$

\section{Intestinal obstruction may be classified into two types:}

- Dynamic- in which peristalsis is working against a mechanical obstruction. It may occur in an acute or a chronic form. It includes intraluminal (faecal impaction, foreign bodies, bezoars, gallstones), intramural (stricture and malignancy) and extramural causes (bands/adhesions, hernia, intususcception and volvulus).

- Adynamic- in which there is no mechanical obstruction; peristalsis is absent or inadequate (e.g. paralytic ileus, mesenteric ischaemia or pseudo-obstruction). ${ }^{[15]}$

\section{Types of obstruction}

\section{Simple Small Bowel Obstruction:}

Typical cases of simple obstruction may show diffuse bowel loop dilatation with a smooth transition zone or a smooth "beak" at the obstructed site on computed tomography (CT). The bowel wall at the site of obstruction may be minimally thickened or of normal thickness. Mesenteric changes, such as vascular engorgement and haziness, are absent or minimal, and ascites is either absent or minimal.

\section{Closed-Loop Obstruction:}

The most important CT indicators may include the whirl sign, convergence of mesenteric vessels toward the twisted site, and reversed position of the mesenteric artery and vein. The whirl sign, however, is also seen in asymptomatic subjects or in patients who had undergone gastric surgery for gastric pathologies.

\section{Strangulated Small Bowel Obstruction:}

Strangulation implies interference with the blood supply associated with an obstruction that may not necessarily be complete.

CT criteria for strangulated obstruction are as follows:

- Portal or mesenteric venous gas, pneumatosis intestinalis

- Abnormal bowel wall enhancement

- Serrated beak sign

- Unusual mesenteric vascular course

- Diffuse mesenteric vascular engorgement and haziness

- Bowel wall thickening

- A large amount of ascites. ${ }^{[16]}$

\section{Subjects and Methods}

\section{Study design}

A prospective study was done with evaluation of forty patients, clinically suspected of intestinal obstruction who were referred to the department of radiodiagnosis.

All patients (age >18yrs) who were clinically suspected for intestinal obstruction with complications (such as strangulation and bowel ischemia) and without complications whose follow up data (surgical) was available were included in the study.

Patients with age <18yrs, deranged renal function tests, allergic to contrast, pregnancy and conservatively managed patients were excluded.

Protocol:

Patients clinically suspected for intestinal obstruction and referred to the department were subjected to CT examination which was performed by using Somatom Scope (Siemens, Erlangen, Germany). The scanning parameter were $130-150 \mathrm{mAs}, 130 \mathrm{kVp}, 6 \times 2.0 \mathrm{~mm}$ collimation, $5 \mathrm{~mm}$ slice thickness, $1.5 \mathrm{~mm}$ reconstruction intervals.

Oral positive/neutral/negative contrast were given in selected patients.

CT images were obtained after administering $100 \mathrm{~mL}$ of intravenous contrast (contrapaque-containing iohexol equivalent to $300 \mathrm{mg}$ iodine, $1.2 \mathrm{mg}$ tremathine, $0.1 \mathrm{mg}$ edetate calcium sodium, water for inj q.s.)

All the patients who underwent CT examination were followed up for surgical management. Correlation of the CT findings with operative findings were done.

\section{Results}

In the present study, the maximum number of patients presenting with intestinal obstruction were in the age group of $31-40$ years i.e. 10 patients $(25 \%)$. The youngest patient in the present series was 19 years old whereas the oldest patient was 90 years old [Table 1].

Out of 40 patients, the number of male patients was more than female patients. $24(60 \%)$ male patients were part of the study, whereas $16(40 \%)$ patients were females on X-ray abdomen (Erect) air-fluid levels were seen in $29(72.50 \%)$ patients. Whereas no air- fluid levels were seen in 11 patients $(27.50 \%)$

In the present study on intestinal obstruction, the level of obstruction was diagnosed in the small bowel in $30(75 \%)$ patients. The level of obstruction was diagnosed in large bowel in $10(25 \%)$ patients Ileum was the most common site of obstruction in the present study. Out of the total 40 patients $13(32.50 \%)$ had distal ileal obstruction. Proximal ileal obstruction was seen in $8(20 \%)$ patients. Jejunal obstruction was seen in $4(10 \%)$ patients and duodenum obstruction was seen in $2(5 \%)$ patients. Ascending/ transverse/ descending colon was the site of obstruction in $7(17.5 \%)$ patients. Rectosigmoid was the site of obstruction in $3(7.5 \%)$ patients. No definite site of obstruction was seen in three patients amongst which two patients who had multiple dense adhesions hence zone of transition was not determined and another patient had prominent distal ileal loops with no narrow zone of transition.

On MDCT, out of total 40 patients, adhesions were found to be the cause of obstruction in $21(52.50 \%)$ patients. Hernia was the cause in $3(7.50 \%)$ patients. Intussusception was the reason of obstruction in $2(5 \%)$ patients. Malignancy was the cause of obstruction in $5(12.5 \%)$ patients. Malrotation was the cause in $4(10 \%)$ patients and malignancy was the cause of obstruction in $5(12.50 \%)$ patients. Ischaemia, intraluminal and indeterminate were the cause in $1(2.5 \%)$ patient each while extrinsic compression was the cause in 2 $(5.00 \%)$ patients.

In the present study on 40 patients with intestinal 
obstruction, small bowel faeces sign (SBFS) was seen in 6 patients $(15 \%)$. Small bowel faeces sign was not seen in 32 patients $(85 \%)$.

Pneumatosis intestinalis was seen in $4(10 \%)$ patients who presented with intestinal obstruction. Rest of the $36(90 \%)$ patients did not have pneumatosis intestinalis.

MDCT correctly diagnosed the cause of obstruction in 39 $(97.50 \%)$ patients. In one patient the cause of dilated gut could not be diagnosed due to microscopic mesenteric carcinomatous deposits in patient with known case of carcinoma ovary.

Adhesion was found to be the most common cause of obstruction on MDCT i.e in 21 patients, however on operative findings only 17 patients were diagnosed with adhesions as the cause [Figure 1,2]. In the rest 4 patients, two patients had pseudo-obstruction i.e no narrow zone of transition was found and whole of the small bowel was dilated. It was proposed that vasospasm as the cause of obstruction while the rest of the two patients had passable stricture in the distal ileum at the surgery.

Hernia was the cause in 3 patients in which one patient had strangulated indirect inguinal hernia while one patient had spigelian hernia and the last one had non obstructed indirect inguinal hernia. [Figure $3 \& 4$ ]

Malignancy was the cause of obstruction in 5 patients. On histopathological examination, malignant growth was adenocarcinoma in 5 patients [Figure 5].

Intussusception was the reason of obstruction in 2 patients. One patient had jejuno-ileal intusussception with meckel's diverticulum as lead point and also ileo-ileal intussusceptions [Figure 6]. Another patient had ileo-ileal intussusception.

Malrotation was given as cause of obstruction in 4 patients. However, only two patients had malrotation while in rest of the patients, one patient had a stricture at the level of jejunoileal junction which was misdiagnosed as malrotation and another patient had no obstruction at surgery, however on X-ray multiple air-fluid levels were observed hence a possibility of intermittent volvulus was considered Ischaemia was due to SMA thrombosis.
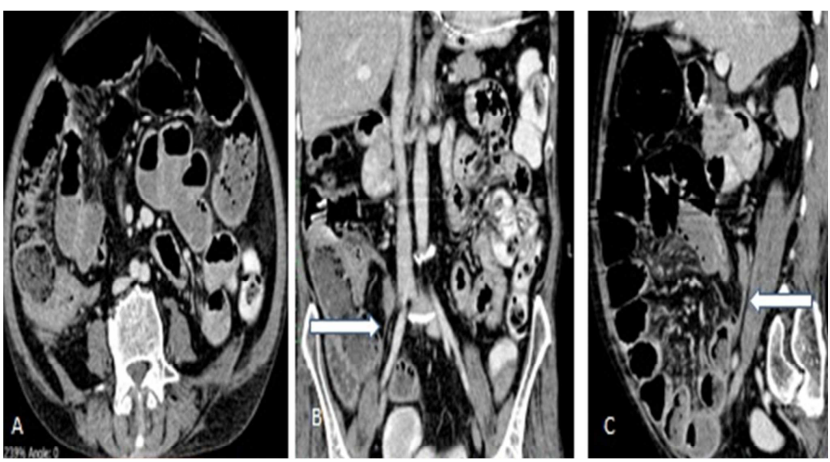

Figure 1: A (axial), B (coronal), C (sagittal) images show a short segment narrow zone of transition in the distal ileal loop with proximal dilatation of the small bowel loops - likely stricture.

Intraluminal cause was found in one patient who had enterolith while extraluminal cause were observed in two patients who had extrinsic compression of the 3rd part of duodenum (superior mesenteric artery syndrome)due to reduced distance and angle between superior mesenteric artery and aorta.

On correlation of MDCT findings with operative findings, $p$ value was 0.00 (significant) and cohen's kappa coefficient value in cases of adhesions was 0.75 (fair correlation), 0.5 in malrotation, 0.68 in malignancy while in rest of the causes it was 1 (perfect correlation).
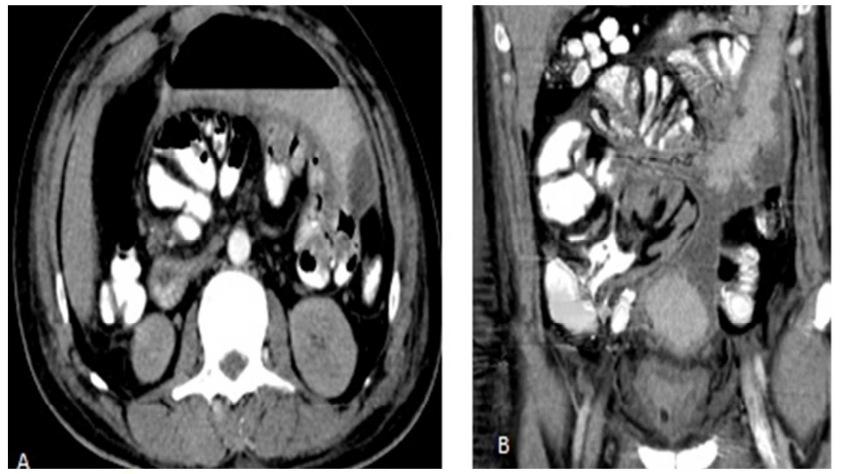

Figure 2: A (axial) and B (coronal) volume rendered CECT abdomen images show matted small bowel loops encased within the thick enhancing membranes forming abdominal coccon with dilated small bowel loops.
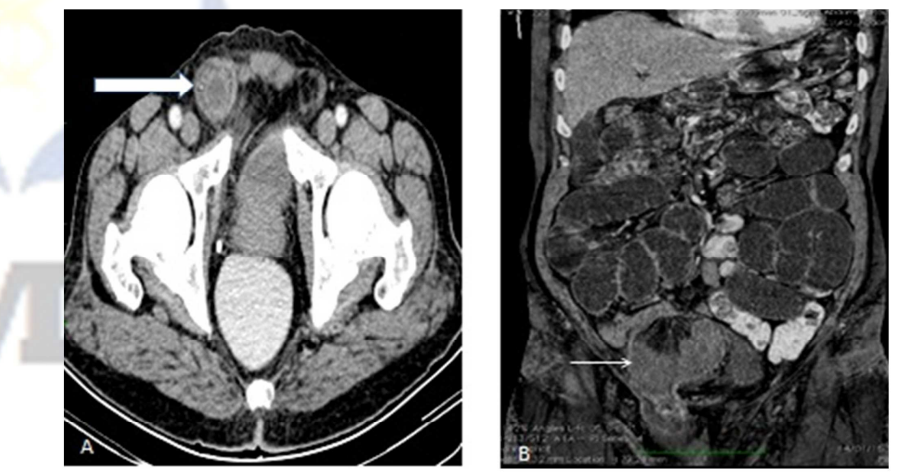

Figure 3: A (Axial) \& B (Coronal) volume rendered reformatted images show right inguinal hernia. Note is made of few hypodense air foci (arrows) in the wall of herniated loop -pneumatosis intestinalis
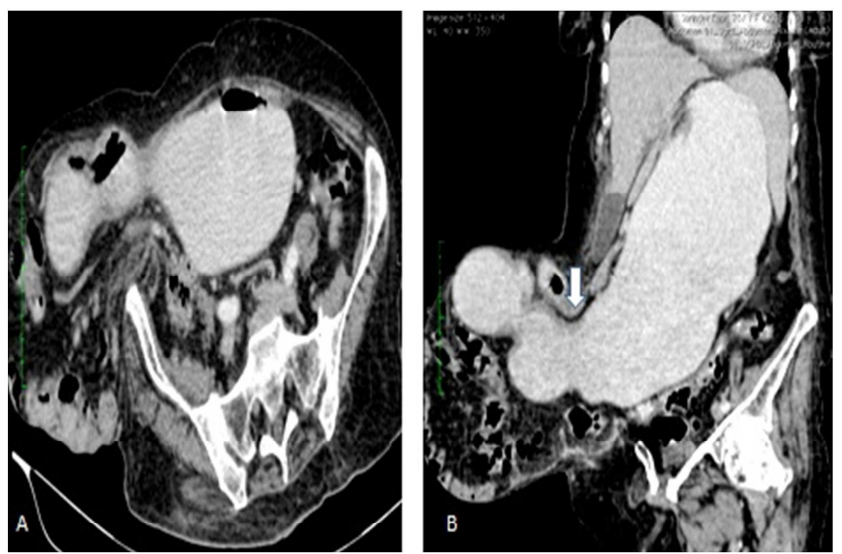

Figure 4: A (axial) and B (coronal) CECT abdomen images show herniation of the stomach and gut loops through abdominal wall. 

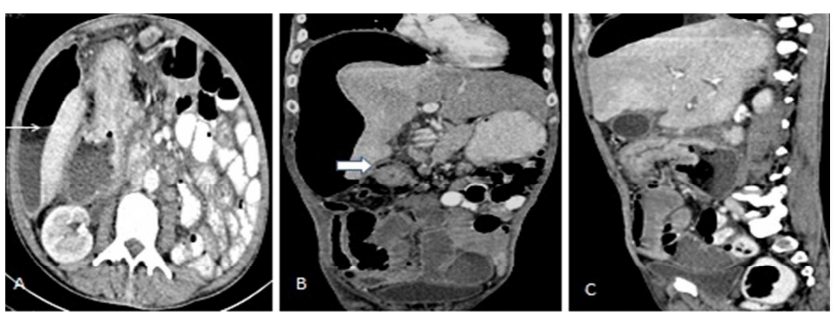

Figure 5: A (axial), B(coronal) and $\mathrm{C}$ (Sagittal) images of CECT abdomen show irregular circumferential enhancing thickening of the ascending colon reaching upto the hepatic flexure with multiple air foci in the wall of ascending colon s/o pneumatosis intestinalis (block arrow). Also a sealed off collection with air fluid level is seen in right paracolic space (arrow)

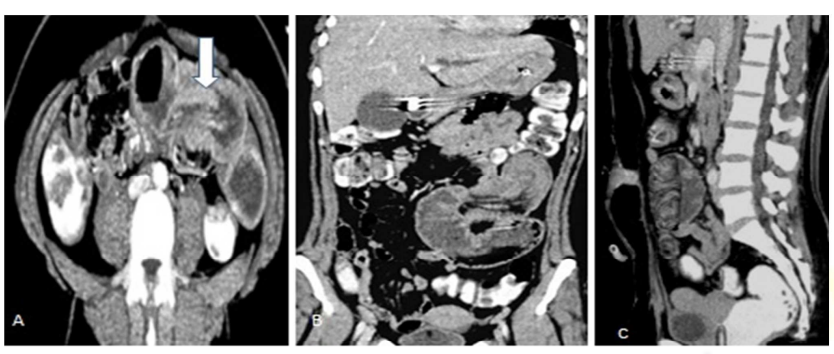

Figure 6: A (Axial), B(Coronal) Contrast enhanced CT images show ileo-ileal intussuception in the left lumbar region.

Table 1: Age Distribution of 40 Patients with Intestinal Obstruction

\begin{tabular}{|c|c|c|}
\hline Age group (Years) & No. of Patients & Percentage \\
\hline$<20$ & 4 & $10 \%$ \\
\hline $21-30$ & 4 & $10 \%$ \\
\hline $31-40$ & 10 & $25 \%$ \\
\hline $41-50$ & 06 & $15 \%$ \\
\hline $51-60$ & 5 & $12.5 \%$ \\
\hline $61-70$ & 7 & $17.5 \%$ \\
\hline$>70$ & 4 & $10 \%$ \\
\hline Total & 40 & 100 \\
\hline
\end{tabular}

\section{Discussion}

The analysis of age distribution in the present study showed a wide range with the maximum number of patients i.e. 10 $(25 \%)$ in the age group of 31-40 years. The youngest patient in the present series was 19 years old, whereas the oldest patient was 90 years old. The results of the present study are in accordance with the study conducted by Saini DK et al in which they stated there was a wide range of age distribution of patients who presented with intestinal obstruction. The most common age group of patients presenting with intestinal obstruction was $30-40$ years $(33 \%)$. ${ }^{[1]}$

On analysing the gender distribution of patients with intestinal obstruction in the present study, it was found that there were more male patients (24) than female (16). Hence the present study is in accordance with the study conducted by Saini DK et al who stated in their study that the number of male patients were more than female i.e. 27 males and 13 females. ${ }^{[17]}$

In the present study, air-fluid levels were seen in 29 $(72.50 \%)$ patients. No evidence of air fluid levels was seen in $16(17.50 \%)$ patients. This is in concordance with study Saini DK et al who studied 40 patients with intestinal obstruction and found that air- fluid levels were seen in $57.50 \%$ patients. ${ }^{[17]}$

In the present study, 30 patients $(70 \%)$ were diagnosed with small bowel obstruction and 10 patients (30\%) had large bowel obstruction which is in concordance to with Megibow et al who conducted a study on sixty four patients with the diagnosis of intestinal obstruction. Among these patients, 55 patients $(86 \%)$ had small bowel obstruction, whereas 9 patients (14\%) had large bowel obstruction. ${ }^{[18]}$

In the study conducted by Suri $\mathrm{S}$ et al, the most common site of obstruction was found to be ileum. ${ }^{[19]}$

Out of total of 40 patients, the level of obstruction was correctly interpreted in 39 patients. In one patient, there was prominence of gut loops, on surgery microscopic metastatic deposits were the cause of obstruction, therefore the site of intestinal obstruction was indeterminate. This is in concordance with study of Megibow et al who retrospectively evaluated $84 \mathrm{CT}$ scans from patients referred for intestinal obstruction. 64 patients were ultimately proved to have intestinal obstruction. CT diagnosed the presence of obstruction in 60 patients $(94 \%)$ and cause of obstruction was diagnosed in 47 patients $(73 \%) .{ }^{[18]}$

The most common site of obstruction was ileum in 21 patients. Distal ileum was the site of obstruction in 13 patients and proximal ileum in 8 patients. The site of obstruction was identified in jejunum in 4 patients and duodenum in 2 patients. Ascending/ transverse/ descending colon was the site of obstruction in 7 patients. Rectosigmoid was identified as the site of obstruction in 3 patients.

Maglinte DD et al retrospectively studied 78 patients with suspected intestinal obstruction. In their study, CT revealed the cause of obstruction in $95 \%$ (39/41) of those patients in whom CT correctly showed the obstruction. ${ }^{[20]}$

Incidence of detection of cause of obstruction in present study was $97.50 \%$ comparable with the study done by Maglinte DD et al in which incidence of detection of cause of obstruction was $95.12 \%$. $^{[20]}$

In the present study, most common cause of the obstruction diagnosed on MDCT was adhesions in 21 patients $(32.50 \%)$, however on operative findings only 17 patients were diagnosed with adhesions as the cause. In the rest 4 patients, two patients had pseudo-obstruction i.e no narrow zone of transition was found and whole of the small bowel was dilated [Figure 8]. It was proposed that vasospasm as the cause of obstruction while the rest of the two patients had passable stricture in the distal ileum at the surgery.

In 4 patients diagnosed on MDCT as malrotation, only two patients had malrotation however in rest of the patients, one patient had a stricture at the level of jejunoileal junction which was misdiagnosed as malrotation and another patient had no obstruction at surgery, however on X-ray multiple air -fluid levels were observed hence a possibility of intermittent volvulus was considered.

In rest of the patients with causes of hernia, intussusception, malignancy, intraluminal and extrinsic compression, MDCT was able to diagnose the findings very accurately and the findings well correlated with the operative findings.

In the present study most common cause of intestinal 


\section{Singh et al; Rale of MDCF in Intestinal Olstruction}

obstruction was adhesions $(32.50 \%)$. The result of present study matched with studies done by Malik AM et al in which commonest cause of obstruction was adhesions comprising $41 \%$ of patients. ${ }^{[21]}$

Malik AM et al evaluated 229 patients with acute intestinal obstruction. Post operative adhesions accounted for $41 \%$ (n $=95$ ) of the total cases, followed by abdominal tuberculosis $(25 \%, \mathrm{n}=58)$, obstructed/ strangulated hernias of different types $(18 \%, \mathrm{n}=42)$. The most common cause of intestinal obstruction was postoperative adhesions. ${ }^{[21]}$

Limitations of my study were small sample size, inadequate distention of the bowel loops with oral/rectal contrast may mimic stricture and oral contrast given in some patients hindered with the detection of the bowel wall thickness and detection of pneumatosis intestinalis.

\section{Conclusion}

MDCT by using its multiplanar reformatting and 3D capabilities is highly specific and accurate in detecting the presence of intestinal obstruction and can demonstrate the exact site of obstruction in high percent of cases and detection of complications with high precession helps in appropriate management of the patient.

\section{References}

1. Khurana B, Ledbetter S, McTavish $\mathrm{J}$ et al. Bowel obstruction revealed by multidetector CT. AJR Am J Roentgenol. 2002;178:113944.

2. Mullan C.P, Siewert B and Eisenberg R.L. Small bowel obstruction. AJR Am J Roentgenol. 2012;198(2):W105.

3. Lappas JC, Reyes BL, Maglinte DD. Abdominal radiography findings in small-bowel obstruction: relevance to triage for additional diagnostic imaging. AJR Am J Roentgenol. 2001; 176(1):167-74.

4. Nicolaou S, Kai B, Ho S et al. Imaging of acute small-bowel obstruction. AJR Am J Roentgenol. 2005;185(4):1036-44.

5. Thompson WM, Kilani RK, Smith BB et al. Accuracy of abdominal radiography in acute small-bowel obstruction: does reviewer experience matter? AJR Am J Roentgenol. 2007; 188(3):W233-8.

6. Lim JH. Intestinal obstruction. In: Maconi G, Porro GB, editors. Ultrasound of the gastrointestinal tract. Berlin, Germany: SpringerVerlag. 2007;27-34

7. Maglinte DD, Howard TJ, Lillemoe KD et al. Small-bowel obstruction state-of-the-art imaging and its role in clinical management. Clin Gastroenterol Hepatol. 2008;6(2):130-9.

8. Shrake PD, Rex DK, Lappas JC et al. Radiographic evaluation of suspected small bowel obstruction. Am J Gastroenterol. 1991; 86(2):175-8.

9. Caoili EM, Paulson EK. CT of small-bowel obstruction: another perspective using multiplanar reformations. AJR Am J Roentgenol. 2000; 174(4):993-8.

10. Maglinte DD, Sandrasegaran K, Lappas JC et al. CT enteroclysis. Radiology2007; 245(3): 661-71

11. Herlinger H, Maglinte DDT. Small bowel obstruction. In: Herlinger H, Maglinte DDT, eds. Clinical radiology of the small intestine. Philadelphia, Pa: Saunders, 1989; 479-507.

12. Ros PR, Huprich JE. ACR Appropriateness Criteria on suspected smallbowel obstruction. J Am Coll Radiol2006; 3(11):838-41.

13. Maglinte DD, Herlinger H, Turner WW et al. Radiologic management of small bowel obstruction: a practical approach. Emerg Radiol1994; 1(13): 138-49.

14. Baker SR. ACR Appropriateness Criteria on small-bowel obstruction: a critique of the term and its terms. J Am Coll Radiol2007; 4(7): 443-5.

15. Evers M. Acute Abdomen. In: Townsend CM, Beauchamp RD, Evers BM, Mattox KL, editors. Sabiston Textbook of Surgery. 19th edition. Philadelphia: Saunders; 2009. p. 1289.

16. Ha HK, Kim JK, Gastrointestinal tract In: Hagga JR, Lanzieri CF, Gilkeson RC editors. CT and MRI of the whole body. 5th edition. Missouri; Mosby;2003; p.1221-2.

17. Saini DK, Chaudhary P, Durga CK et al. Role of multislice computed tomography in evaluation and management of intestinal obstruction. Clinics and Practice. 2013;3(20).51-5.

18. Megibow AJ, Blathzar EJ, Cho KC et al. Bowel obstruction evaluation with CT. Radiology 1991;180:313-8.

19. Suri S, Kaur H, Wih JD et al. CT in abdominal Tuberculosiscomparison with barium studies. Indian J Radiol Imag. 1993;3:237-42.

20. Maglinte DD, Gage SN, Harmon BH et al. Obstruction of small intestine: Accuracy and role of $\mathrm{CT}$ in diagnosis Radiology. 1993:188:61-4

21. Malik AM, Shah M, Pathan R et al. Pattern of acute intestinal obstruction: Is there a change in the underlying etiology? Saudi Journal of Gastroenterology. 2010;16(4): 4-85.

Copyright: () the author(s), publisher. Asian Journal of Medical Radiological Research is an Official Publication of "Society for Health Care \& Research Development". It is an open-access article distributed under the terms of the Creative Commons Attribution Non-Commercial License, which permits unrestricted non-commercial use, distribution, and reproduction in any medium, provided the original work is properly cited.

How to cite this article: Singh A, Makkar IK, Thukral CL, Gupta K, Uppal MS. Intestinal Obstruction: Role of MDCT with Surgical Correlation. Asian J. Med. Radiol. Res. 2018;6(2):12-16.

DOI: dx.doi.org/10.21276/ajmrr.2018.6.2.4 